\title{
Approximation of the IEEE 802.11p Standard Using Commercial Off-The-Shelf IEEE 802.11a Hardware
}

\author{
Wim Vandenberghe, Ingrid Moerman, Piet Demeester \\ Internet Based Communication Networks and Services \\ Ghent University \\ Ghent, Belgium \\ Wim.vandenberghe@intec.ugent.be
}

\begin{abstract}
IEEE 802.11p hardware is hard to find. Previous research efforts often relied on project-specific prototype implementations which are characterized by a high cost and are not always available to the entire research community. Commercially available turnkey implementations are rare and quite expensive compared to commercial of-the-shelf (COTS) IEEE $802.11 \mathrm{a} / \mathrm{b} / \mathrm{g}$ hardware. However, the difference between the IEEE 802.11p amendment and the other IEEE 802.11 standards is quite small. It can be seen as a combination of the IEEE 802.11a and IEEE 802.11e standards, with some specific adjustments. This paper presents how an approximation of the IEEE 802.11p standard can be implemented using COTS IEEE 802.11a hardware and some specific software adjustments. This way, vehicular test infrastructures can be established in a much more cost effective manner, and existing IEEE 802.11 wireless testbeds can be used to support VANET research.
\end{abstract}

Keywords: IEEE 802.11p, ETSI ITS-G5, VANET, FOT

\section{INTRODUCTION}

Many publications in the VANET domain rely on simulations for their research. Often an integration of traffic and network simulators is used because this results in more realistic mobility patterns and VANET connectivity properties. Examples of such simulation frameworks are iTETRIS [1] and VGSim [2]. The main advantage of simulation platforms is the fact that they enable the research of VANETs on a large scale and in a very cost-effective manner. However, there are also limitations regarding simulations of wireless networks: they are not entirely representative for the real-life performance of the same solutions when deployed in realistic environments. This phenomenon can be caused by several factors such as simplified traffic patterns and end-user models, errors introduced by imperfect hardware or software drivers, deviating assumptions about signal propagation and interference, etc. Several studies exist that elaborate these potential problems in more detail [3] - [5]. Therefore, Field Operational Tests (FOTs) are in general considered as a useful means to further validate the simulated research results. However, for researchers it can be quite challenging to establish the test infrastructure for such FOTs. In VANET literature, some experiments with real hardware can be found, but in most cases prototype hardware was used. A well known example of such a prototype is the CVIS communication board which is a PCI card with several sensors and two CALM-M5 modules [6]. In general, prototypes are characterized by a high cost, and are not always available to the entire research community. Hardware that successfully evolved from prototype to commercial hardware hardly exists. At the time of writing and to the best of our knowledge, only one IEEE 802.11p solution is commercially available: the Unex DCMA$86 \mathrm{P} 2$ mini-PCI card [7]. However, this card is sold without a supporting driver, therefore it cannot be seen as a turnkey solution for VANET researchers. Besides, with a price tag of approximately 170 US dollar, it is definitely more affordable than prototype solutions, but compared to commercial of-theshelf (COTS) IEEE $802.11 \mathrm{a} / \mathrm{b} / \mathrm{g}$ hardware it is still quite expensive. Furthermore, existing wireless testbeds are typically equipped with such COTS interfaces, replacing them by the Unex card (which only supports IEEE 802.11p) would limit the usability of the testbed for other domains. From the above, it can be concluded that an approximation of the IEEE $802.11 \mathrm{p}$ standard using COTS IEEE 802.11a hardware and some specific software adjustments would be useful. In this paper it is described how such a solution can be implemented.

\section{DESCRIPTION OF THE IEEE 802.11P STANDARD}

The IEEE $802.11 \mathrm{p}$ standard is designed to enable the deployment of VANETs in high-speed environments. It is an amendment to the IEEE 802.11-2007 standard, and combines the physical layer supplement IEEE 802.11a with the Quality of Service (QoS) amendment IEEE 802.11e.

The PHY layer of the standard uses the same signal processing and specification as the OFDM PHY used in the IEEE 802.11a standard. To achieve a robust connection under high velocities, small modifications are proposed [8], [9]. Instead of using the full clocked mode of $20 \mathrm{MHz}$, the usage of the half clocked mode with $10 \mathrm{MHz}$ bandwidth is foreseen in vehicular environments. Consequently, parameters in the time domain are doubled and data rates are halved. This approach makes the signal more robust: effects of Doppler spread are reduced because of the reduced bandwidth and the larger guard interval reduces inter-symbol interference caused by multi-path propagation.

To provide larger communication ranges for vehicular scenarios, four classes of maximum allowable Effective Isotropic Radiated Power (EIRP) up to $44.8 \mathrm{dBm}(30 \mathrm{~W})$ are defined. The largest value can only be used by emergency 
vehicles; a typical value for safety relevant messages is 33 $\mathrm{dBm}(2 \mathrm{~W})$ with a realistic communication range of 750 meters [9]. These values are significantly higher than the IEEE 802.11a EIRP values used in the United States of America (800 $\mathrm{mW})$ and Europe $(500 \mathrm{~mW})$ [11]. To avoid interference from non-VANET equipment, dedicated operating frequency bands are used. The $5.9 \mathrm{GHz}$ American ITS band divides $75 \mathrm{MHz}$ $(5.850-5.925 \mathrm{GHz})$ into seven $10 \mathrm{MHz}$ channels and a $5 \mathrm{MHz}$ safety margin at the lower end of the band. In Europe, $30 \mathrm{MHz}$ of bandwidth has been reserved for vehicular communication $(5.875-5.905 \mathrm{GHz})$, which is divided into 3 channels. Furthermore, member states are given the possibilities to also reserve the other 4 channels of the American ITS band (5.850 5875 and 5.90 - $5.925 \mathrm{GHz}$ ) on a national level. The European maximum EIRP for ITS is $33 \mathrm{dBm}(2 \mathrm{~W})$. It should be mentioned that these European regulations are implemented by the ETSI ITS-G5 standard, which is based on and very closely related to IEEE 802.11p [9]. In the rest of this paper we will focus on the IEEE standard, and consider all results to be also applicable to the ETSI standard.

The IEEE 802.11p MAC layer is equal to the IEEE 802.11e Enhanced Distributed Channel Access (EDCA) Quality of Service (QoS) extension [12]. This scheme is similar to the standard IEEE 802.11 CSMA/CA scheme called distributed coordination function (DCF), but EDCA can differentiate between 4 different application categories (AC): background traffic (BK), best effort traffic (BE), voice traffic (VO) and video traffic (VI). Different Contention Window (CW) and Arbitration Inter Frame Space (AIFS) values are chosen for the different application categories, where VI has the highest priority and BK the lowest. The values used by IEEE $802.11 \mathrm{p}$ where specifically chosen for vehicular communication scenarios. Other specific parameter values also distinguish the MAC layer of the EEEE 802.11e and IEEE 802.11p standard: in the latter SIFS is $32 \mu$ s and the slot time is $13 \mu$ s.

In the context of vehicular ad hoc networks, IEEE 802.11p is complemented with other standards to cover additional layers in the protocol suite: the IEEE 1609 standards set. Collectively, IEEE 802.11p and 1609.x are called wireless access in vehicular environments (WAVE). IEEE 1609 consists of four documents, from which IEEE 1609.4 is most relevant to this paper since it defines the multi-channel operation of WAVE (that is implemented on top of the MAC layer) [13]. In this scheme, WAVE devices can choose to utilize only one channel (the control channel $\mathrm{CCH}$ ), but they can also alternate between $\mathrm{CCH}$ and a service channel $(\mathrm{SCH})$. WAVE devices can organize themselves in small networks called WAVE basic service sets (WBSS). Based on a synchronization mechanism, channel switching can be performed in such a way that it assures that all WAVE devices are monitoring the $\mathrm{CCH}$ during a common time interval, and that the devices that joined a specific WBSS are utilizing the corresponding $\mathrm{SCH}$ during a common time interval. The sum of both intervals comprises the sync interval. Dimensioning these intervals can influence the available throughput on both channels, and thus also influence latency, scalability and reliability characteristics [14].

\section{APPROXIMATION USING COMMERCIAL OFF-THE-SHELF IEEE 802.11A HARDWARE}

The difference between the IEEE 802.11p amendment and the other IEEE 802.11 standards is quite small. As already comprehensively discussed in section II it can be seen as a combination of the IEEE 802.11a and IEEE 802.11e standards, with some specific adjustments. With this in mind, it should be possible to implement an approximation of the IEEE 802.11p standard using COTS IEEE 802.11a hardware and some specific software adjustments. This way, vehicular test infrastructures can be established in a much more cost effective manner, and existing IEEE 802.11 wireless testbeds can be used to support VANET research. To implement such an IEEE $802.11 \mathrm{p}$ approximation, the differences between the different standards have to be cleared as much as possible. Based on the description given in section II, they are summarized in Table 1. In the next subsections we will describe how every aspect of the IEEE 802.11p approximation was handled in our implementation

\section{A. Reduced channel bandwidth}

The first significant difference between both standards is the fact that the channel width has to be reduced from $20 \mathrm{MHz}$ to $10 \mathrm{MHz}$. As a result, all parameters in the time domain are doubled (symbol duration, guard time, PLCP preamble duration and signal field) and the subcarrier spacing is halved. As a consequence of these changes the available data rates are halved, but the signal becomes more robust under vehicular circumstances. The reduced bandwidth reduces the effects of Doppler spread and the larger guard interval reduces intersymbol interference caused by multi-path propagation.

The simplest way to implement this behavior is to rely on a hardware-driver combination that supports $10 \mathrm{MHz}$ channels natively. A few chipsets exist on the market that allow this, e.g. the Atheros RF5122, RF5133 or AR9390, but they are not widely applied in wireless interface cards. Besides, WLAN testbeds are already operational in several research institutes, the goal of the presented IEEE 802.11p approximation is to be compatible with such existing infrastructures. For instance, the IBBT w-iLab.t testbed which is available to the authors of this paper is an extensive facility consisting of 200 nodes, each

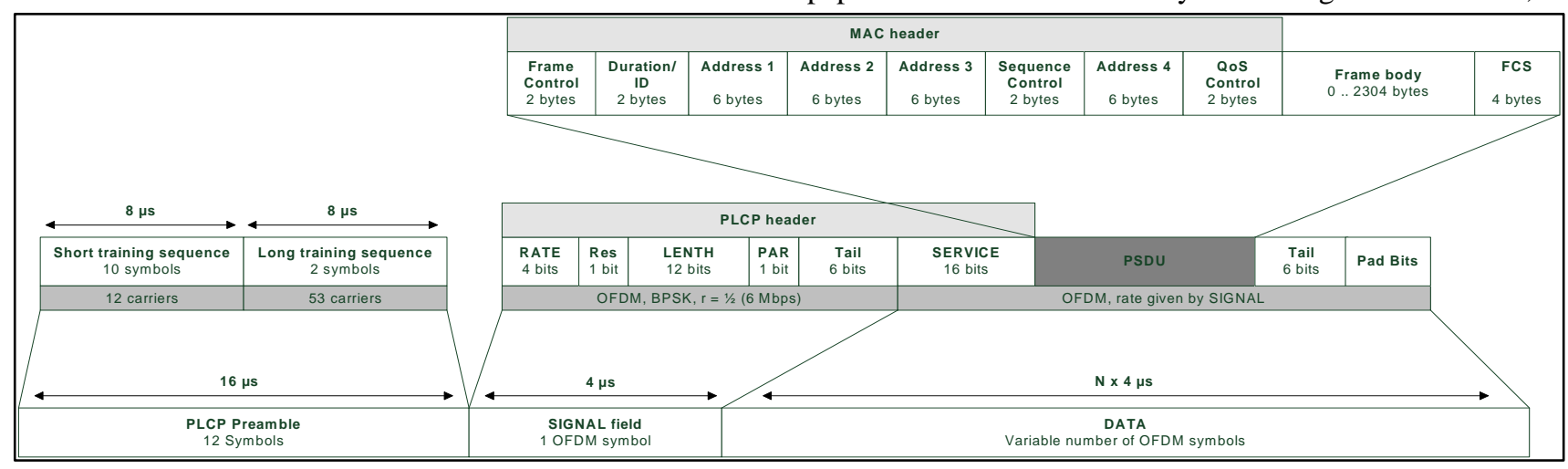

Figure 1: IEEE 802.11 packet format 
equipped with two IEEE $802.11 \mathrm{a} / \mathrm{b} / \mathrm{g}$ network interfaces. A software approximation of the 11p standard on top of this testbed would turn it into a valuable tool for VANET research. The wireless cards used in this testbed are Compex WLM54SAG 200mW IEEE $802.11 \mathrm{a} / \mathrm{b} / \mathrm{g}$ miniPCI cards which are installed on PC Engines Alix 3D3 motherboards. The Compex card uses an Atheros AR 5413 chipset and supports a maximum output power of $200 \mathrm{~mW}(23 \mathrm{dBm})$. It is supported in Linux by the Madwifi and the Ath5k drivers. However, using this card with any of these drivers does not allow switching from $20 \mathrm{MHz}$ channels to $10 \mathrm{MHz}$ channels. Therefore it is not possible to influence the symbol duration, guard time, PLCP preamble duration, signal field duration or the subcarrier spacing. This means that the implemented approximation of the IEEE 802.11p standard will provide a less robust signal under real vehicular circumstances than real IEEE 802.11p hardware. This is an important aspect that should be taken into account during the analysis of experimental results, but it is not an insuperable obstacle because the approximation then represents a worst case scenario. Because it still uses 20 $\mathrm{MHz}$ channels and thus provides a less robust signal, VANET solutions that are validated using this approximation will also work on real hardware with better signal quality. But it is important for experimenters to be aware of this property.

Besides a better signal quality, the $10 \mathrm{MHz}$ channels also lead to a reduction of the available throughput. This is an important aspect for research targeting the network congestion problem in VANETs, and therefore should definitely be implemented in the presented approximation. A simple solution would be just to change the data rate of the wireless card to the specific envisaged scenario. E.g. when the VANET scenario defines a data rate of $6 \mathrm{Mbps}$, just set the IEEE 802.11a interface on the $6 \mathrm{Mbps}$ data rate. The flaw in this approach is the fact that in this case the used modulation on the PHY layer is not correct. At a data rate of $6 \mathrm{Mbps}$, IEEE $802.11 \mathrm{p}$ relies on quadrature phase-shift keying (QPSK) with a $1 / 2$ coding rate, while IEEE 802.11a uses binary phase-shift keying (BPSK) modulation. To guarantee the correct modulation selection for a selected bandwidth, the principle of data padding was adopted in the implementation. The basic concept is that when a packet is to be transmitted, it is extended with meaningless data to guarantee that the transmission duration of the packet is the same as it would be in the IEEE 802.11p standard. After reception of a packet, the padding data is removed before further processing of the packet by the higher network layers. This way, actual throughput for a selected IEEE 802.11a data rate is halved, guaranteeing a correct mapping in the IEEE $802.11 \mathrm{p}$ approximation between selected modulation and available throughput. The downside of this approach is that more symbols have to be communicated correctly between a transmitter and a receiver, what could lead to higher Packet Error Rates (PER) in the approximation than in real IEEE $802.11 p$ hardware. Similar to the usage of a wider channel this ensures that the approximation is a worst-case representation of the actual hardware, and does not interfere with usage of the approximation for real-life validation of VANET solutions. Another specific property of the data padding solution is that less application data can be transmitted since the maximum packet length is determined by the MTU of the IEEE 802.11 interface (2272), and for every application data byte a padding byte is included in the packet. This is not a problem as long as application developers are aware of this limitation. E.g. using our specific IPv6 VANET networking approach presented in previous work [15] together with standard UDP, the application data limit per packet is 1000 bytes when using the maximum MTU. This is sufficient, since al common scenarios for Cooperative Systems rely on cooperative awareness messages (CAM) and decentralized environment notification messages (DENM) which both have a packet size of about 300 bytes [16] - [18].

Several aspects are taken into account when determining the correct amount of padding bytes. As depicted in Figure 1, from a data rate point of view the transmission of a packet consists of three steps. The first one is the transmission of the PLCP Preamble with a fixed duration. Since the transmission time for this preamble is $16 \mu$ s longer in the IEEE $802.11 p$ standard than in the IEEE 802.11a standard, a certain number of padding bytes is required to mimic this behavior. The second step is the transmission of the SIGNAL field, which is one OFDM symbol that always relies on BPSK modulation with a $1 / 2$ coding rate $(4 \mu$ s in IEEE $802.11 \mathrm{a})$. The third step is the transmission of the rest of the packet at the selected data rate. To extend the packet transmission duration with $20 \mu \mathrm{s}$ to take care of the first two steps, it is required to enlarge the packet with enough bytes to require transmission of 5 extra OFDM symbols. Because the amount of data that can be

Table 1: Differences between IEEE 802.11a/e and IEEE 802.11p/CALM-M5

\begin{tabular}{|l|l|l|}
\hline Parameter & IEEE 802.11 a / e & IEEE 802.11 p / CALM-M5 \\
\hline Channel width & $20 \mathrm{MHz}$ & $10 \mathrm{MHz}$ \\
\hline Date rates & $6,9,12,18,24,36,48,54 \mathrm{Mbit} / \mathrm{s}$ & $3,4.5,6,9,12,18,24,27 \mathrm{Mbit} / \mathrm{s}$ \\
\hline Symbol duration & $4 \mu \mathrm{s}$ & $8 \mu \mathrm{s}$ \\
\hline Guard time & $0.8 \mu \mathrm{s}$ & $1.6 \mu \mathrm{s}$ \\
\hline PLCP Preamble duration & $16 \mu \mathrm{s}$ & $32 \mu \mathrm{s}$ \\
\hline Signal Field duration & $4 \mu \mathrm{s}$ & $8 \mu \mathrm{s}$ \\
\hline Subcarrier spacing & $0.3125 \mathrm{MHz}$ & $0.15625 \mathrm{MHz}$ \\
\hline Frequency range & USA: $5.15-5.35 \mathrm{GHz} \& 5.725-5.825 \mathrm{GHZ}$ & $\begin{array}{l}\text { USA: } 5.850-5.925 \mathrm{GHz} \\
\text { EU: } 5.875-5.905 \mathrm{GHz} \& \text { optionally other } \\
\text { channels of USA band }\end{array}$ \\
\hline $\begin{array}{l}\text { Maximum EIRP } \\
\text { EU: } 5.15-5.35 \mathrm{GHz} \& 5.47-5.725 \mathrm{GHz}\end{array}$ & $\begin{array}{l}30 \mathrm{~W} \text { (emergenc y vehicle in USA) } \\
2 \mathrm{~W} \text { (normal vehicle US A \& EU) }\end{array}$ \\
\hline SIFS duration & $\begin{array}{l}\text { USA: } 800 \mathrm{~mW} \\
\text { EU: } 500 \mathrm{~mW}\end{array}$ & $32 \mu \mathrm{s}$ \\
\hline Slot time & $16 \mu \mathrm{s}$ & $13 \mu \mathrm{s}$ \\
\hline $\begin{array}{l}\text { CWMin (V0, VI, BE, } \\
\text { BK) }\end{array}$ & $4 \mu \mathrm{s}$ & $3,3,7,15$ (CCH channel) \\
\hline $\begin{array}{l}\text { CWMax (VO, VI, BE, } \\
\text { BK) }\end{array}$ & $7,15,1023,1023$ & $7,7,15,1023$ (CCH channel) \\
\hline $\begin{array}{l}\text { AIFSN (VO, VI, BE, } \\
\text { BK) }\end{array}$ & $2,2,3,7$ & $2,3,6,9$ (CCH channel) \\
\hline $\begin{array}{l}\text { Multi-Channel operation } \\
\text { Not supported }\end{array}$ & $\begin{array}{l}\text { US: Channel switching single radio } \\
\text { EU: One radio CCH + one radio SCH }\end{array}$ \\
\hline
\end{tabular}


transmitted using a single symbol is dependant of the chosen modulation parameters, the amount of required padding data is different for every data rate. The actual values are summarized in Table 2. To handle the third step, it is required to add a number of padding bytes that is equal to two bytes for the PLCP SERVICE field plus the length of the PSDU (which includes the QoS aware MAC header, the frame body and the frame check sequence) plus one byte for the tail bits.

Table 2: Required amount of padding bytes for the preamble and signal fields

\begin{tabular}{|l|l|l|l|}
\hline $\begin{array}{l}\text { Data rate } \\
\text { (Mbps) }\end{array}$ & $\begin{array}{l}\text { Data bits } \\
\text { per symbol }\end{array}$ & $\begin{array}{l}\text { Data bytes } \\
\text { per symbol }\end{array}$ & $\begin{array}{l}\text { Padding } \\
\text { bytes }\end{array}$ \\
\hline 6 & 24 & 3 & 15 \\
\hline 9 & 36 & 4.5 & 22.5 \\
\hline 12 & 48 & 6 & 30 \\
\hline 18 & 72 & 9 & 45 \\
\hline 24 & 96 & 12 & 60 \\
\hline 36 & 144 & 18 & 90 \\
\hline 48 & 192 & 24 & 120 \\
\hline 54 & 216 & 27 & 135 \\
\hline
\end{tabular}

\section{B. Frequency range and maximum EIRP}

Besides the time domain differences induced by halving the used channel bandwidth, the differences in frequency range and maximum EIRP between the IEEE 802.11a and IEEE 802.11p standard have to be handled in the presented approximation. The regulations for .11a in the United States of America and the European Union [19] are given in Table 3. Regarding IEEE 802.11a usage in the EU, Transmit Power Control (TPC) is required if the EIRP is higher than $50 \mathrm{~mW}$ or the frequency is higher than $5.25 \mathrm{GHz}$. If TPC is not implemented, the maximum EIRP given in Table 3 should be halved.

Table 3: IEEE 802.11a EIRP regulations

\begin{tabular}{|l|l|l|l|}
\hline Region & Frequency & Placement & EIRP \\
\hline USA & $5.15-5.25 \mathrm{GHz}$ & Indoor & $40 \mathrm{~mW}$ \\
\hline USA & $5.25-5.35 \mathrm{GHz}$ & $\begin{array}{l}\text { Indoor \& } \\
\text { outdoor }\end{array}$ & $200 \mathrm{~mW}$ \\
\hline USA & $5.725-5.825 \mathrm{GHz}$ & Outdoor & $800 \mathrm{~mW}$ \\
\hline EU & $5.15-5.35 \mathrm{GHz}$ & Indoor & $200 \mathrm{~mW}$ \\
\hline EU & $5.47-5.725 \mathrm{GHz}$ & $\begin{array}{l}\text { Indoor \& } \\
\text { outdoor }\end{array}$ & $1000 \mathrm{~mW}$ \\
\hline
\end{tabular}

As already mentioned, the implementation presented in this paper targets usage on COST Compex WLM54SAG 200mW IEEE $802.11 \mathrm{a} / \mathrm{b} / \mathrm{g}$ miniPCI wireless interfaces. Therefore, when using these cards the most suitable available values are $5.825 \mathrm{GHz}$ at $200 \mathrm{~mW}$ in the USA, and $5.700 \mathrm{GHz}$ at $200 \mathrm{~mW}$ in the EU. In the frequency domain, the difference between these values and the actual IEEE 802.11p frequencies $(5.850-$ $5.925 \mathrm{GHz}$ ) seems negligible in terms of radio signal behavior (propagation, multipath distortion, etc.). The difference in max EIRP however is more significant, and should be taken into account when defining experiments and analyzing results. The available communication range will be significantly less using the approximation since the maximum EIRP of $200 \mathrm{~mW}$ is an order of magnitude smaller than the $2 \mathrm{~W}$ typically used in vehicular scenarios. This property of the approximation can be overcome by scaling the inter-vehicle distances appropriately to achieve the same number of vehicles in a collision domain as in the actual scenarios. This requires knowledge about the real life link characteristics of the implemented approximation of IEEE 802.11p. This will be discussed in section IV.

\section{EDCA functionality}

On the MAC layer, the IEEE 802.11e Enhanced Distributed Channel Access (EDCA) Quality of Service (QoS) extension has to be included in the approximation of the IEEE 802.11p standard. This can be achieved using a device driver that implements EDCA. Two possible candidates in combination with the envisaged COTS Compex hardware are the Madwifi and the Ath5k drivers [20]. Since the EDCA support was just introduced recently in the Ath5k driver, it was chosen to use the less experimental Madwifi driver which already provides this support for a longer time. The Madwifi driver allows to change the EDCA parameter settings using the Linux "iwpriv" command. This way the approximation can use the actual EDCA parameters of the IEEE 802.11p standard. It should be mentioned that this command actually sets the ECWMin and ECWMax parameters instead of the CWMin and CWMax values given in Table 1 , but the correct values can easily be translated using the following formulas:

$$
\begin{aligned}
& \text { CWMin }=2^{\text {ECWMin }}-1 \\
& \text { CWMax }=2^{\text {ECWMax }}-1
\end{aligned}
$$

Some other MAC parameters in the time domain should be adjusted to mimic IEEE $802.11 \mathrm{p}$ behavior. The slot time should be changed from 4 to $13 \mu \mathrm{s}$. Using the Madwifi driver, this can easily be achieved by changing the value found in the file "/proc/sys/dev/wifiX/slottime". The other parameter that should be changed is the SIFS duration: it should become $32 \mu \mathrm{s}$ instead of $16 \mu \mathrm{s}$. Directly changing this value using the Madwifi driver turned out to be not possible. In the source code of the driver a static function was found that defines this value, but after changing it no actual change in behavior could be experimentally shown. It is likely that this value is also determined in the closed source binary HAL that is part of the Madwifi driver, or that it is defined on the hardware itself. However, an indirect manner was found to implement this change. SIFS is used in two ways: as the small time interval between the data frame and its acknowledgement and between an RTS and CTS; and as a parameter value to determine the Arbitration Interframe Space as follows:

$$
\text { AIFS }=\text { SIFS }+(\text { AIFSN } x \text { slot duration })
$$

In a vehicular environment where most information will be locally broadcasted, both acknowledgments and RTS/CTS packets will not be utilized and can therefore be neglected in the approximation of the IEEE 802.11p standard. This means that the goal of extending the SIFS duration actually is to extend the AIFS duration, in this case with $16 \mu \mathrm{s}$. This goal can however also be achieved by raising the AIFSN value. This can be done using the Madwifi driver. Because the slot duration is $13 \mu \mathrm{s}$, the AIFSN values will all be raised with one for all QoS classes. Consequently, the AIFS values of the approximation will be $3 \mu$ s less than in the IEEE 802.11p standard. This can 
be neglected since this ranges between $2 \%$ and $5 \%$ of the total AIFS duration for the different QoS classes. The actual values to be used by the "iwpriv" command to approximate IEEE 802.11p using IEEE 802.11a hardware and the Madwifi drivers are given in Table 4.

Table 4: "iwpriv" parameters to approximate .11p using .11a hardware

\begin{tabular}{|l|l|l|l|}
\hline AC & ECWmin & ECwmax & AIFSN \\
\hline VO & 2 & 3 & 3 \\
\hline VI & 2 & 3 & 4 \\
\hline BE & 3 & 4 & 7 \\
\hline BK & 4 & 10 & 10 \\
\hline
\end{tabular}

\section{Disabled beaconing}

An important aspect of the presented approximation is the requirement that it should be guaranteed that only packets generated by the ITS applications or VANET routing protocols are transmitted. This means that all standard beaconing (which normally occurs when configuring an IEEE 802.11 wireless network interface in infrastructure or ad-hoc mode) should be disabled. Using the Madwifi driver, this behavior can be accomplished by configuring the interface in a special mode called monitor mode. In this case it does not perform any beaconing, but the interface just listens on a given channel, and transmits packets that are given to it by Linux processes. This way, total control of all transmitted packets is given to the application and routing protocol developers. It has to be mentioned that this mode expects raw packets. This means that before handing a packet over to the wireless interface all headers (IP header, MAC header, 802.11, etc) should be correctly encapsulated on the packet. Although this results in a light overhead for the developers of VANET routing protocols, it has the important advantage that such raw packets contain valuable information such as RSSI values, channel frequency, data rate, etc. It also enables the definition of both transmission power and rate per transmitted packet. Handling raw packets, transferring them from and to wireless interfaces in monitor mode can be easily done using the Click Modular Router [21]. This is a modular software router platform originally developed by MIT with subsequent development by a broad research community. It is the ideal platform for efficient prototyping of VANET networking protocols using the implemented approximation of the IEEE 802.11p standard. All developments on the networking layer that were done in the scope of this paper were implemented in this framework.

\section{E. Multi-channel operation}

On top of the IEEE 802.11p MAC layer, the IEEE 1609.4 standard defines the multichannel operation mode of IEEE $802.11 \mathrm{p}$ where the radio interface continuously alternates between the control channel and a service channel. The advantage of this approach is that even cheaper single-radio devices can use both safety and comfort services. The downside is that the available throughput in the control channel (where the important safety messages are all communicated) is halved. Other drawbacks are synchronized frame collisions and bandwidth wastage as analyzed by Campolo [14]. Therefore in the EU, the current consensus is to use two distinct radio interfaces where one is always tuned to the control channel and the other can switch to the appropriate service channel as announced on the control channel. Keeping the known VANET scalability issues in mind, the authors of this paper are convinced that the single-channel per interface operation is the more appropriate method. Therefore multi-channels per interface is not supported in the proposed solution.

\section{EXPERIMENTAL CHARACTERIZATION OF IMPLEMENTATION}

To gain insights into the actual performance of the presented IEEE 802.11 approximation, a measurement campaign was performed with two vehicles in line of sight highway conditions. This public highway consists of two lanes in every direction, divided by a concrete crash barrier. It is part of the R4 ringway around the city of Ghent, Belgium. The already mentioned hardware was used: Compex WLM54SAG 200mW IEEE $802.11 \mathrm{a} / \mathrm{b} / \mathrm{g}$ miniPCI cards installed on PC Engines Alix 3D3 motherboards. The dual band antennas of these wireless interfaces were connected to a magnetic base placed on the roof of the vehicle. One vehicle was parked in an inlet at the side of the highway, in a $90^{\circ}$ angle compared to the highway orientation. The other vehicle passed at a constant speed of $80 \mathrm{~km} / \mathrm{h}$. The EU settings were applied as described in subsection B $(5.700 \mathrm{GHz}$ at $200 \mathrm{~mW})$, application packet size was 40 bytes. An external GPS receiver was used to determine the distance between sender and receiver during the measurements. Several test runs were combined in a single dataset to eliminate abnormal behavior that could be caused by specific circumstances due to the other traffic. The results are depicted in Figure 2, indicating a range of approximately 450 meters in highway line-of-sight conditions.

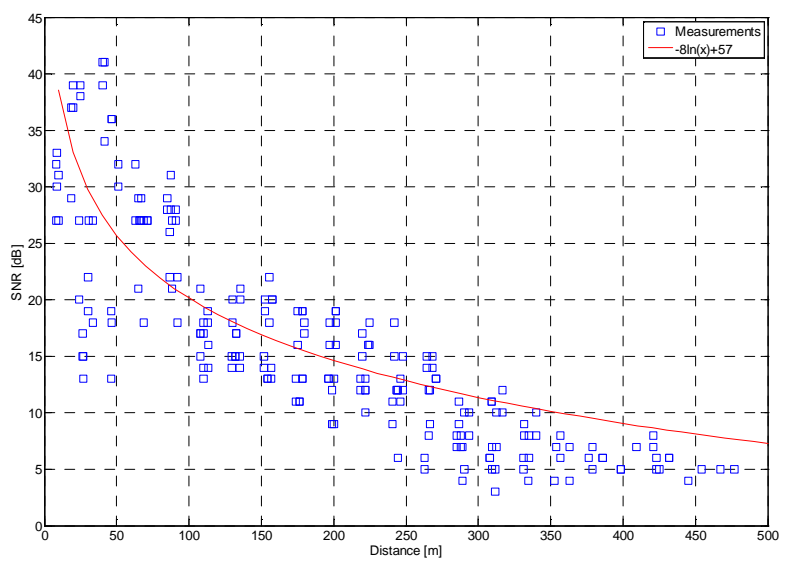

Figure 2: Communication range in highway environment

It should be mentioned that experimental results based on the presented work can also be used as an input to improve and validate simulator studies. As an example we use the above results to determine a suitable path loss model for VANET simulations. Such a model can have a significant influence on simulated results. The model of Kunish [22] is one of the possible candidates for application in VANET simulations. In Figure 2, a data fitting function was added. As can be seen in Figure 3, this trendline closely corresponds with the Kunish model, confirming its suitability for VANET simulations. 


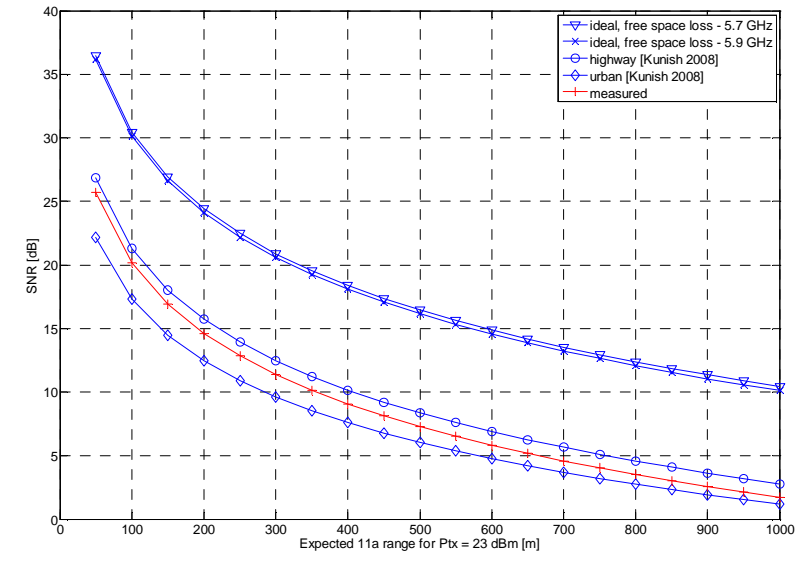

Figure 3: Comparison of literature path loss models with experimental measurements

\section{CONCLUSION}

In this paper it was investigated if an approximation of the IEEE 802.11p standard can be implemented using COTS IEEE 802.11a hardware and some specific software adjustments. Several differences could indeed be eliminated through software changes: several MAC parameters could be adjusted flawlessly (e.g. CWMin, CWMax, AIFS, slottime), and uncontrolled beaconing was entirely disabled. Other differences were approximated in such a way that the introduced deviations of the .11p standard can be neglected (e.g. transmission frequency, SIFS duration, the omitted multichannel behavior and the available throughput for a given datarate). Some aspects however could not be approximated: the channel bandwidth remains $20 \mathrm{MHz}$ instead of the desired $10 \mathrm{MHz}$, and the maximum allowable Effective Isotropic Radiated Power (EIRP) remains $200 \mathrm{~mW}$ instead of $2 \mathrm{~W}$. Under real vehicular circumstances this results in a less robust signal and a lower communication range than real IEEE 802.11p hardware. However, this is not an insuperable obstacle because the approximation represents a worst case scenario. VANET solutions that are validated using this approximation will also work on real hardware with better signal quality. As long as experimenters are aware of the limitations of the presented solution, it can be successfully applied as a cost-effective tool for VANET research.

\section{REFERENCES}

[1] V. Kumar, R. Bauza, F. Filali, J. Gozalvez, L. Lin, M. Rondinone, "iTETRIS - A Large Scale Integrated Simulation Platform for V2X Communications. Application to Real-Time Traffic Management", in Proc of 9th International Conference on Intelligent Transport System Telecommunications (ITST), Lille, 2009

[2] B. Liu, B. Khorashadi, H. Du, D. Ghosal, C.-N. Chuah, M. Zhang, "VGSim: An Integrated Networking and Microscopic Vehicular Mobility Simulation Platform", IEEE Communications Magazine, Vol 47, Issue 5, 2009

[3] D. Kotz, C. Newport, R. S. Gray, J. Liu, Y. Yuan, C. Elliot, "Experimental Evaluation of Wireless Simulation Assumptions", in Proceedings of the 7th ACM international symposium on Modeling, analysis and simulation of wireless and mobile systems (MsWiM), Venice, 2004

[4] D. Cavin, Y. Sasson, A. Schiper, "On the Accuracy of Manet Simulators", in Proc of the second ACM international workshop on Principles of mobile computing, New York, 2002
[5] J. Heidemann, N. Bulusu, J. Elson, C. Intanagonwiwat, K.-C. Lan, Y. $\mathrm{Xu}$ et al., "Effects of Detail in Wireless Network Simulation", in Proc. Of SCS Communication Networks and Distributed Systems Modeling and Simulation Conference, 2001

[6] K. Sjöberg et al., "Measuring and using the RSSI of 802.11p", in Proc. ITS World 2010, Busan, Korea, 2010

[7] Unex, "DCMA-86P2: industrial grade, high power $5.86 \sim 5.92 \mathrm{GHz}$ wifi mini-PCI module for $802.11 \mathrm{p} / \mathrm{DSRC}$ application, AR5414A-B2B" http://www.unex.com.tw/product/dcma-86p2, last accessed 16/5/2011

[8] IEEE WG802.11 - Wireless LAN Working Group, “ 802.11 p-2010 IEEE Standard for Local and Metropolitan Area Networks - Specific requirements Part 11: Wireless LAN Medium Access Control (MAC) and Physical Layer (PHY) Specifications Amendment 6: Wireless Access in Vehicular Environments", http://standards.ieee.org, 2010

[9] ETSI, "ETSI ES 202663 Intelligent Transport Systems (ITS); European profile standard for the physical and medium access control layer of Intelligent Transport Systems operating in the $5 \mathrm{GHz}$ frequency band", http://www.etsi.org, 2010

[10] L. Stibor, Y. Zang and H.-J. Reumerman, "Evaluation of communication distance of broadcast messages in a vehicular ad-hoc network using IEEE 802.11p", in Proc. Wireless Communications and Networking Conference (WCNC), Hong Kong, 2007, pp. 254-257.

[11] IEEE Computer Society, "IEEE Std 802.11tm-2007 IEEE Standard for Information technology - Telecommunications and information exchange between systems - Local and metropolitan area networks Specific requirements - Part 11: Wireless LAN Medium Access Control (MAC) and Physical Layer (PHY) Specifications", http://standards.ieee.org, 2007

[12] IEEE Computer Society, "IEEE Std 802.11etm-2005 IEEE Standard for Information technology - Telecommunications and information exchange between systems - Local and metropolitan area networks Specific requirements - Part 11: Wireless LAN Medium Access Control (MAC) and Physical Layer (PHY) Specifications- Amendment 8: Medium Access Control (MAC) Quality of Service Enhancements", http://standards.ieee.org, 2005

[13] IEEE 1609 WG, "1609.4-2010 - IEEE Draft Standard for Wireless Access in Vehicular Environments (WAVE) - Multi-channel operation", http://standards.ieee.org, 2010

[14] C. Campolo, Y. Koucheryavy, A. Molinaro, A. Vinel, "On the performance of beaconing in $802.11 \mathrm{p} /$ WAVE Vehicular Ad Hoc Networks", in Proc. 10th International Conference on ITS Telecommunications (ITST), Kyoto, Japan, 2010

[15] W. Vandenberghe, E. Van de Velde, D. Carels, J. Bergs, C. Blondia, I. Moerman, P. Demeester, "VANET addressing scheme incorporating geographical information in standard IPv6 header", Proc. 10th International Conference on ITS Telecommunications (ITST), Kyoto, Japan, 2010

[16] ETSI, "ETSI TR 102638 Intelligent Transport Systems (ITS); Vehicular Communications; Basic Set of Applications; Definitions", http://www.etsi.org, 2009

[17] ETSI, "ETSI TS 102 637-2, Intelligent Transport Systems; Vehicular Communications; Basic Set of Applications; Part 2: Specification of Cooperative Awareness Basic Service",http://www.etsi.org, 2010.

[18] ETSI, "ETSI TS 102 637-3, Intelligent Transport Systems; Vehicular Communications; Basic Set of Applications; Part 2: Specification of Decentralized Environment Notification Basic Service", http://www.etsi.org, 2010.

[19] J. Schiller, "Mobile communications 02 Edition", published by Pearson Eductation Limited, 2003.

[20] “The madwifi project", http://madwifi-project.org/, , last accessed online $16 / 5 / 2001$

[21] E. Kohler, "The Click modular router", $\mathrm{PhD}$ thesis, Massachusetts Institute of Technology, 2000

[22] J. Kunisch, J. Pamp, "Wideband Car-to-Car Radio Channel Measurements and Model at 5.9 GHz,", Proc. Vehicular Technology Conference, 2008. VTC 2008-Fall. IEEE 68th, vol., no., pp.1-5, 21-24 Sept. 2008 